\title{
An Ontology-Based Integrated Approach to Situation Awareness for High-Level Information Fusion in C4ISR
}

\author{
María-Cruz Valiente $^{1}$, Rebeca Machín ${ }^{2}$, Elena García-Barriocanal ${ }^{1}$, \\ and Miguel-Ángel Sicilia ${ }^{1}$ \\ ${ }^{1}$ Computer Science Department, University of Alcalá, \\ Ctra. Barcelona km. 33.6 - 28871 Alcalá de Henares (Madrid), Spain \\ \{maricruz.valiente, elena.garciab, msicilia\}@uah.es \\ 2 AMPER Programas, \\ Ctra. de Andalucía, km 12.700, 28906 Getafe (Madrid), Spain \\ rmachineamper.es
}

\begin{abstract}
Sharing of situation awareness is considered a fundamental capability for C4ISR systems, including decision support systems. However, the current evolution of information and communication technology stress this need even further, because of the fast changing environment and the need to perceive, analyse and understand a huge amount of data. The adoption of adequate tools and technologies to increase proper situation awareness is required. The SAWCORE Ontology formalizes the knowledge associated to situation awareness enabling operators to increase good decision-making and good performance. On the other hand, the JC3IEDM is a data model aimed at providing interoperability among heterogeneous $\mathrm{C} 2$ systems. In this context, this paper presents the integration of the SAW-CORE Ontology and the JC3IEDM OWL Ontology using mappings in order to provide high-level information fusion for situation awareness in C4ISR systems. This allows us to approach C4ISR situation awareness with reasoning capabilities from an ontological perspective in military missions. For this purpose, SAW-CORE constructs are integrated into the JC3IEDM ontological constructs.
\end{abstract}

Keywords: C4ISR, Situation Awareness, Ontology, JC3IEDM, OWL, SRWL.

\section{Introduction}

The ever changing environment of the Command, Control, Communications, Computers, Intelligence, Surveillance and Reconnaissance (C4ISR) domain, in particular, military missions specially in coalition operations, requires operators to adopt new technologies so they can successfully face the rapid changes that take place and make correct decisions. Therefore, shared relevant situation awareness information is considered to be a critical issue in C4ISR decision-making. Situation awareness entails a broad range of information about objects and items, and their relationships, so the monitoring and management of associated knowledge for successful mission execution is of major importance. Furthermore, situation awareness is represented at Level 2 (Situation Assessment) of information fusion in the Joint Directors of Laboratory 
(JDL) data fusion model (Fig. 1). Since military missions have to deal with information originated from various independent origins, data fusion is a critical task in their systems. Steinberg et al. [1] define situation assessment as "estimation and prediction of relations among entities, to include force structure and cross force relations, communications and perceptual influences, physical context, etc."

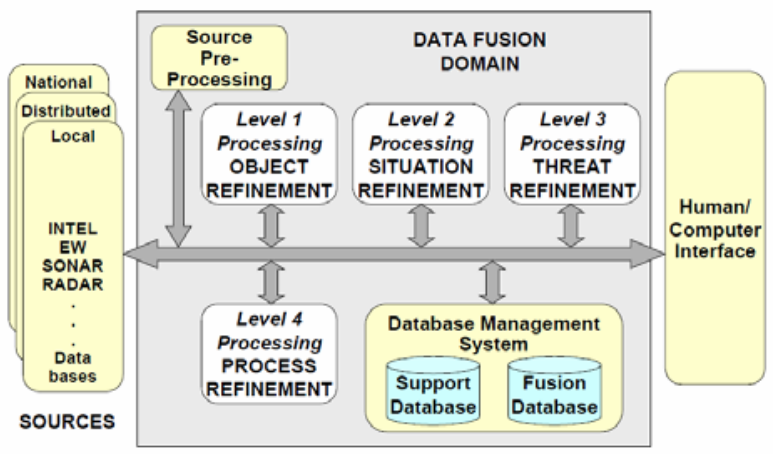

Fig. 1. JDL data fusion model [1]

In the C4ISR domain, the Joint Consultation, Command and Control Information Exchange Data Model (JC3IEDM) [2] is broadly used as an information exchange data model for C4ISR systems. In short, the JC3IEDM specifies the minimum data set required for interoperability between Command and Control Information Systems (C2ISs) and the information exchange mechanism. A C2IS is a way by which military organizations implement command and control.

In another direction, the Situation Awareness Core Ontology (hereinafter, the SAW-CORE Ontology) [3] formalizes the main concepts of situation awareness using a language that can be processed and supported by computer tools. In this sense, the SAW-CORE Ontology is an approach to capture the knowledge associated with situation awareness that supports high-level reasoning. That is, the SAW-CORE Ontology represents a unifying theory of situation awareness compatible with current thinking about situation awareness in military scenarios.

In this paper, we propose an ontology-based situation awareness model that integrates the concepts of the SAW-CORE Ontology into the elements of the JC3IEDM OWL Ontology described by Matheus and Ulicny [4] using mappings.

The rest of this paper is structured as follows. Section 2 covers the background of situation awareness in the C4ISR domain, including a brief explanation of the JC3IEDM model. Section 3 briefly introduces the SAW-CORE Ontology, describing the main elements of the ontology, whereas situation awareness in the JC3IEDM OWL Ontology is described in Section 4. In Section 5, it is explained in detail how SAW-CORE constructs can be integrated into the JC3IEDM OWL constructs in order to provide situation awareness improvement for high-level information fusion in C4ISR systems. Finally, we conclude in Section 6 with some final remarks and future work. 


\section{Background}

\subsection{Situation Awareness for C4ISR}

Key to efficient and safe cooperation is to share a jointly maintained, accessible and reliable situation awareness picture. The operational approach to build requires for each party involved to provide its local situational data, have fusion of them and dissemination of the consolidated result to the parties involved in the area. The information involved can be either the outcome of human field observations or sensor data.

In the recent years, situation awareness has started facing the challenge of shifting the research focus from Level 1 to Level 2 in the JDL data fusion model [1]. In a broad sense, situation awareness can be defined "as the perception of the elements in the environment within a volume of time and space, the comprehension of their meaning and the projection of their status in the near future" [5]. The statement "within a volume of time and space" contained in the definition of situation awareness pertains to the fact that operators constrain the parts of a situation that are of interest to them, based on not only space (i.e., how far away an specific element is), but also how soon that element will have an impact on the operator's goals and tasks. Perception of time and the temporal dynamics associated with events play represents key components of situation awareness in several domains [6].

Although the concepts related to situation awareness vary widely depending on the domain, situation awareness principles and the mechanisms used for achieving situation awareness can be described generically [6]. For example, three levels of situation awareness have been identified in [6]: (i) Level 1-Perception: this level represent the perception of relevant information to form correct pictures of the situation under study; (ii) Level 2 - Comprehension: this level represents the integration of multiple pieces of information and a determination of their relevance to the operator's goal; and (iii) Level 3 - Projection: this level represents the ability to forecast future situation events and dynamics. This ability to project from current events and dynamics to anticipate future events (and their implications) allow for timely decision-making. The dynamic nature of situations dictates that as the situation is always changing, so the operator's situation awareness must constantly change or be rendered out-dated and thus inaccurate.

\subsection{The JC3IEDM}

Although the JC3IEDM is intended first and foremost for the exchange of command, control and communication information between information systems, it nowadays serves more and more as the basis for the general data models that underlie C2ISs. Its evolution has been governed by formal statements of operational information exchange requirements supplemented by the knowledge of domain experts. In this evolution of the model, the information structure is defined in terms of a data model that specifies the basic elements to obtain interoperability between C2ISs. The JC3IEDM does not impose any restriction and, therefore, each organization can adapt it to their own specific circumstances. 
The Multilateral Interoperability Programme (MIP) ${ }^{1}$ in conjunction with the North Atlantic Treaty Organization (NATO) ${ }^{2}$ is the configuration manager of JC3IEDM specifications. The JC3IEDM is included into NATO STANAG 5525. Fig. 2 illustrates the common core of data to be exchanged when using the JC3IEDM information exchange data model [7].

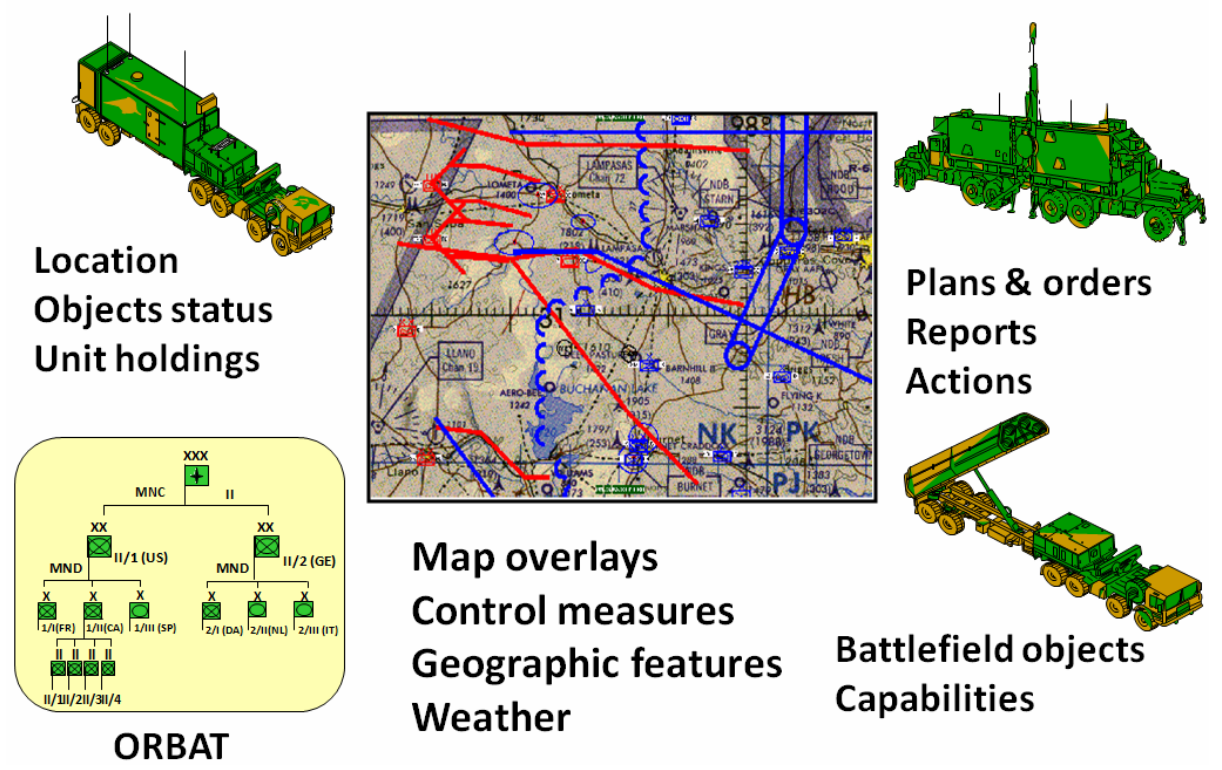

Fig. 2. Operational awareness as depicted in [7]

The JC3IEM specification is a relational schema that was developed using the IDEF1X ${ }^{3}$ notation and it makes also a $\mathrm{UML}^{4}$ notation version available by conversion of IDEF1X. IDEF1X represents a method for designing relational databases with a syntax designed to support the semantic constructs necessary to develop a conceptual schema.

The JC3IEDM model is aimed at monitoring activities with some objective to meet. In the JC3IEDM model, situation awareness is the basis for planning the use of the available resources in activities that lead to the achievement of objectives. Once the missions are planned, they are turned into orders for execution. Execution needs to be monitored to determine the effects achieved. This, in turn, modifies the current situation on which the next mission planning cycle is based. For this purpose, the first requirement to define is the objects that will be managed. The five basic categories of objects include facilities, features, materiel, organisations and persons. Once the

\footnotetext{
${ }^{1}$ https://mipsite.lsec.dnd.ca/

${ }^{2} \mathrm{http}: / /$ www.nato.int/

${ }^{3} \mathrm{http}: / /$ www.idef.com/

${ }^{4}$ http://www.omg.org/
} 
objects of interest are defined, then their location, state and relationships between them can be specified [7].

Situation awareness in the JC3IEDM model provides information relating to objects and may encompass many kinds of information. For example, knowledge derived from relevant events, recorded in the data model, is part of the situation awareness. The main objective is to capture all time-dependent or dynamic properties of interest (for example, the operational status of the positioned units or their current spatial and temporal localization) with the exception of planned activities which are separated from situation awareness. Although events are described structurally in the same way as plans and orders (i.e., planned activities), they are separated because events belong conceptually to situation awareness [7].

There are five independent entities in the JC3IEDM model that could be used for situation awareness, as shown in Fig. 3.

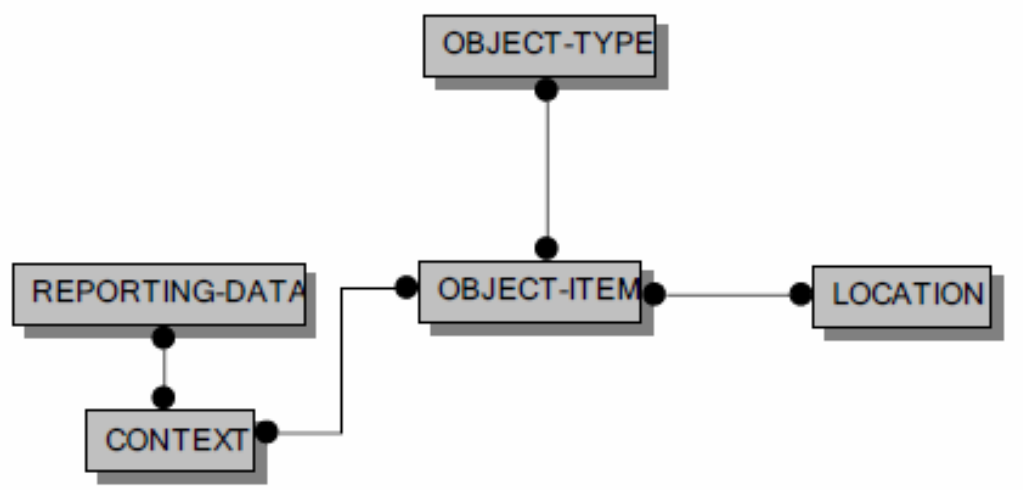

Fig. 3. JC3IEDM entities for Situation Awareness (adapted from [8])

\section{The SAW-CORE Ontology}

In the work presented in [3], Matheus et al. proposed the SAW-CORE Ontology for modelling situation awareness. the SAW-CORE Ontology is a formal approach, encoded in OWL [9], for reasoning about situations, which represents objects, relationships and their evolution over time. The main elements of the SAW-CORE Ontology has demonstrated its capability to support situation modelling. The main elements of the SAW-CORE Ontology are depicted in Fig. 4.

The Situation class defines a situation as a collection of Goals, SituationObjects and Relations. The SituationObject class represents entities in a situation (physical and abstract) that can have characteristics (i.e., Attributes) and can participate in relationships (i.e., Relations). The Attribute class defines values of specific object characteristics, such as weight or colour. The PhysicalObject class is a special type of SituationObject that defines the Volume, Position and Velocity of a specific object. The Relation class define the relationships between ordered sets of SituationObjects. Each relation may be defined according to a set of rules (using the Rule class) involving situation awareness. That is, relations combine pairs of situation objects with truth 
values defined over time by the firing of Rules that define the Relations. For example, as described by Matheus et al. [10], a part at a facility is classified as 'critical' if the current demand at the facility exceeds the current local supply, and it is classified as 'marginal' if the total 'resuppliable rate' for the part at the facility is below a requiredsurplus threshold.

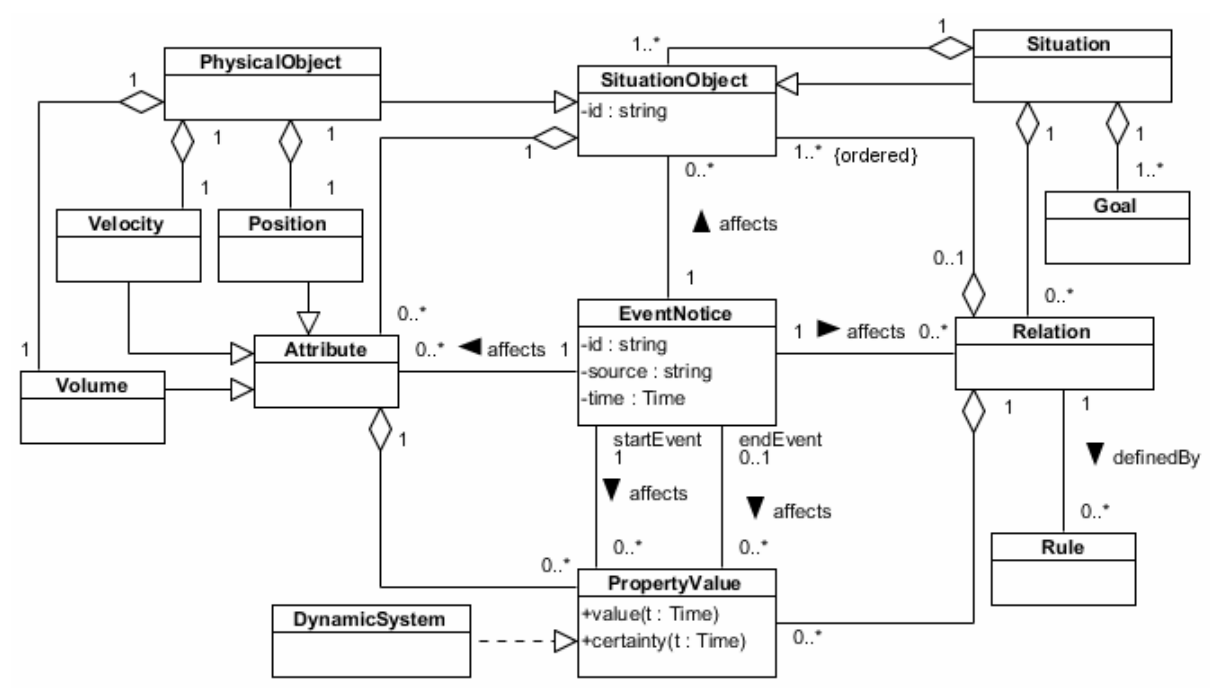

Fig. 4. UML class diagram representing the SAW-CORE ontology (adapted from [3])

An important aspect of Attributes and Relations is that they need to be associated with values that can change over time. To do so, Attributes/Relations are associated with zero or more PropertyValues each of which defines two time-dependant functions, one for the actual value and the other for the certainty assigned to that value. A new PropertyValue is created for an Attribute/Relation whenever an EventNotice arrives that affects that Attribute/Relation. The value of an Attribute/Relation at a particular point in time (current, past or future) can be determined by accessing the value function of the PropertyValue instance that is in effect at the prescribed time. The EventNotice class contain information about events in the real-world situation observed by a sensory source at a specific time that affects a specific Relation or Attribute (of a specific SituationObject) by defining or constraining its PropertyValue. These are the entities that indicate change in the situation and thus are the vehicles by which changes are affected in the Attributes and Relations of the situation representation. The ontology permits a PropertyValue to be implemented as a DynamicSystem. What this means is the value and certainty functions are dynamically modelled and therefore they cause the PropertyValue to change even in the absence of new EventNotices. For example, it is reasonable to assume that an object continues to move with its last reported speed and direction until informed otherwise. To be able to make such projections in the absence of explicit sensory information predictive models are required. It is for this reason that the SAW-CORE Ontology shows DynamicSystems as a way of implementing PropertyValues. Certain attributes, such as Position, would 
be modelled by dynamic systems that might themselves generate internal EventNotices to update the attribute values, with some lesser degree of certainty, until new external sensory information arrives.

\section{The JC3IEDM OWL Ontology for Situation Awareness}

VIStology ${ }^{5}$ developed a JC3IEDM OWL Ontology that comprises over 7900 elements (OWL classes, properties and their instances) from the JC3IEDM ERWIN specification [4]. The JC3IEDM OWL Ontology can act as the basis for providing situation awareness in C4ISR systems.

Five named element groups classify all of the elements of interest [4]: (i) Entity_Group: this group includes specific entities that will become OWL classes along with their corresponding Attribute_Group; (ii) Attribute_Group: this group contains the specific attributes (OWL object properties or OWL datatype properties) for the corresponding entities; (iii) Relationship_Group: this group includes the relationships that can occur between entities, each of which will be turned into OWL object properties; (iv) Domain_Group; and (v) Validation_Rule_Group. The Domain_Group in conjunction with the Validation_Rules_Group contains the allowed values for the domains and ranges of the attributes. Many of the attributes in the JC3IEDM range over values that are codes having corresponding text descriptions of their meanings. These codes are organized into domains that have associated validation rules to define the set of valid values. Codes are captured within enumeration classes within OWL.

Many of the entities exist as sub-classes of at most one other entity. However, not all entities are defined as OWL classes, only those that have additional attributes or relations appear as OWL classes. All others are represented by values of categorycodes. A category-code value is a string that is used to identify a sub-class of a specific OWL class. OWL classes that have sub-classes defined in this way will have an OWL object property whose name is the class name in lowercase with the string 'category-code' added to it. For example, the OWL object property reporting-datacategory-code for the class REPORTING-DATA (domain of the property). Furthermore, there will be a range for this OWL object property with the same name. For example, the OWL class ReportingDataCategoryCode is the range of the OWL object property reporting-data-category-code with the next values: 'ASS' (Assumed), 'ERR' (Erroneous), 'INFER' (Inferred), 'PLAN-6' (Planned), 'PRDCTD' (Predicted) and 'REP-1' (Reported). Fig. 5 shows an excerpt of the JC3IEDM OWL Ontology involving situation awareness.

The CONTEXT class is defined as a collection of information that provides in its entirety the circumstances, conditions, environment, or perspective for a situation in a particular mission (operation). Operators can use the context information to judge the merits of an operational plan, and make changes in order to respond to a changing operational situation. The CONTEXT class can also be used to record the history of an evolving operation, capture a situation, as it existed at some time in the past, or portray a situation, as it is expected to exist at a future date [2]. The CONTEXT-ELEMENT class is part of a CONTEXT and contains information about all REPORTING-DATAs that are

\footnotetext{
${ }^{5}$ http://vistology.com/
} 
part of a specific operation. The REPORTING-DATA class is the specification of source, quality and timing that applies to reported data [2]. The CONTEXT-ELEMENT-STATUS class keeps track of the inclusion status and its timing. This technique preserves data integrity while allowing the data content to change for any instance of CONTEXT [2].

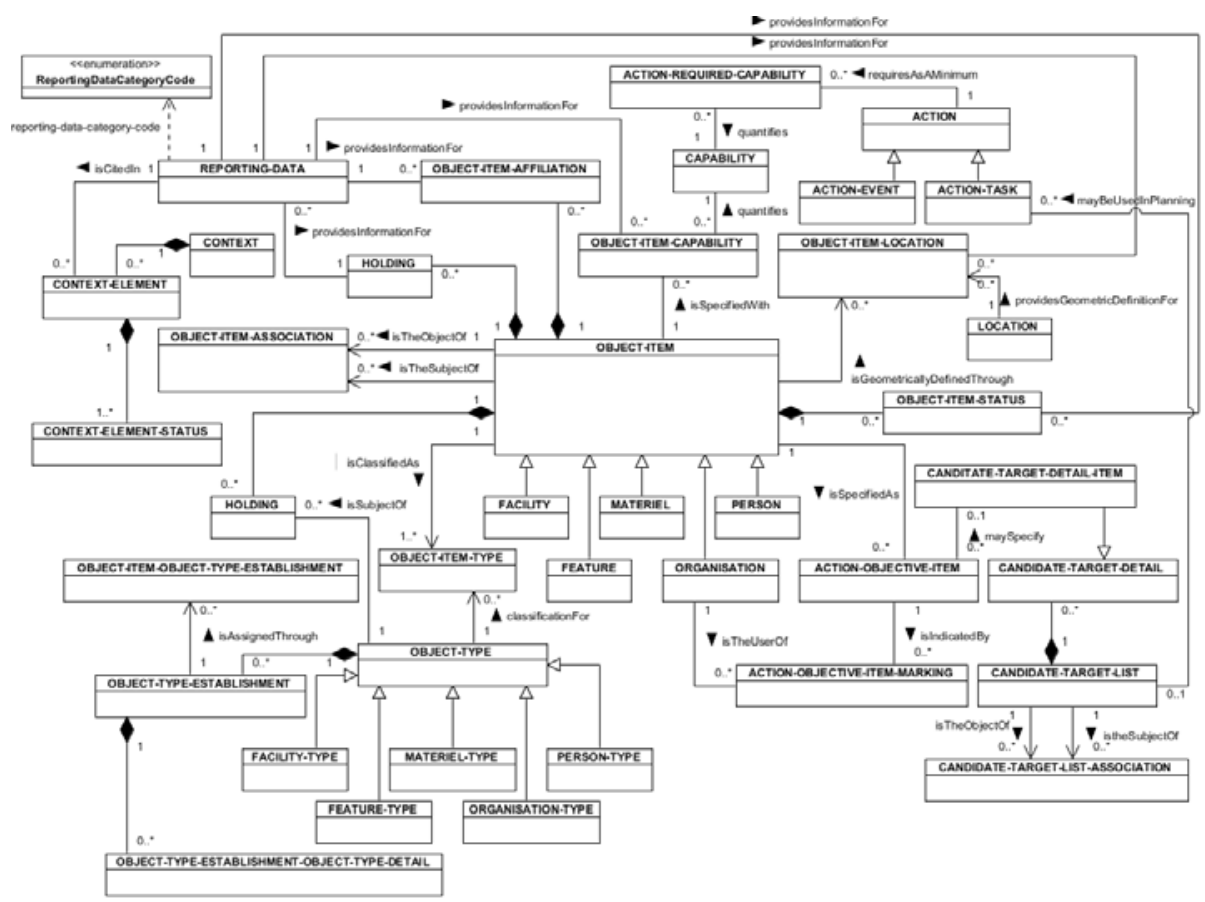

Fig. 5. UML class diagram representing an excerpt of the JC3IEDM OWL Ontology for situation awareness

The objectives of an operation are captured by the ACTION-OBJECTIVE-ITEMMARKING and CANDIDATE-TARGET-LIST classes. The ACTION-OBJECTIVEITEM-MARKING class represents the technique of indicating the position of an ACTION-OBJECTIVE-ITEM at a given time for the benefit of a using ORGANISATION. This class is used to specify requirements, plans, and results of marking an ACTION-OBJECTIVE-ITEM position or an associated reference position [2]. The ACTION-OBJECTIVE-ITEM-MARKING class provides an opportunity to add coordinating details for the user of the marking services. On the other hand, the CANDIDATE-TARGET-LIST class represents a list of selected battlespace objects or types that have potential value for destruction or exploitation, for consideration in planning battlespace activities. That is, the CANDIDATE-TARGET-LIST class is aimed at enabling the maintenance of target lists for consideration during planning processes [7]. A CANDIDATE-TARGET-LIST is composed of CANDIDATE-TARGETDETAILs that itemise candidate targets individually. The CANDIDATE-TARGETDETAIL-ITEM class is a CANDIDATE-TARGET-DETAIL that may be specified as an ACTION-OBJECTIVE-ITEM. Since target lists are often likely to be related to 
each other, such as battalion and brigade-nominated lists with division lists, the model includes the CANDIDATE-TARGET-LIST-ASSOCIATION class.

In the JC3IEDM OWL Ontology, a full characterization of an individual thing includes both item and type attribution. Where the attributes are placed is a design decision [7]. In this way, FACILITY-TYPE, FEATURE-TYPE, MATERIEL-TYPE, ORGANISATION-TYPE and PERSON-TYPE are the classes that represent the OBJECT-TYPEs in the JC3IEDM OWL Ontology (that is, classes of objects that have military or civilian significance for situation awareness), whereas FACILITY, FEATURE, MATERIEL, ORGANISATION and PERSON are the classes that represent OBJECT-ITEMs (that is, individually identified objects that has military or civilian significance for situation awareness). The OBJECT-ITEM-TYPE class represents the assignment type classification to an OBJECT-ITEM (i.e., an OBJECT-ITEM is classified as one or more OBJECT-ITEM-TYPEs using the OBJECT-ITEM-TYPE class).

Another important aspect of situational awareness in the JC3IEDM OWL Ontology is the relationship between items and types that are part of an operation. The OBJECT-ITEM-ASSOCIATION class associates OBJECT-ITEMs with other $O B J E C T$-ITEMs in the sense of belonging, using, controlling, being constrained by, occupying, etc. For example, a division has full command of three brigades, or full command of two and operational control of the third [7]. The HOLDING class specifies what an OBJECT-ITEM actually has or is estimated to have at a particular time. The HOLDING class associates a specific object with a class of objects where the general notion of inclusion has the sense of ownership, possession, assignment, or control, that is who has what at which time. This is an accounting of available assets. One of the purposes of HOLDING is to provide operators with a dynamic update of changes to information on stockpiles of equipment personnel and consumable held by forces [7]. Establishments are specifications that associate, under specified conditions for a given instance of a type of object, a number of instances of other object types as its constituent elements. Essentially, it is a statement of composition of a type of object [7]. The OBJECT-TYPE-ESTABLISHMENT class collects all the components that make up the composition through the detail entity. An instance of OBJECT-TYPE has zero, one or more OBJECT-TYPE-ESTABLISHMENTs each of which consists of a number of instances of other $j c 3: O B J E C T-T Y P E$ s that are listed as instances of jc3:OBJECT-TYPE-ESTABLISHMENT-OBJECT-TYPE-DETAIL. Furthermore, instances of establishments are assigned to instances of OBJECT-ITEM by means of the OBJECT-ITEM-OBJECT-TYPE-ESTABLISHMENT class.

Finally, OBJECT-ITEM-AFFILIATION, OBJECT-ITEM-CAPABILITY, OBJECTITEM-LOCATION and OBJECT-ITEM-STATUS are other OWL classes that we use in our context of situation awareness in C4ISR.

The OBJECT-ITEM-AFFILIATION class is refers to geopolitical, ethnic, religious, or functional preferences of a specific OBJECT-ITEM. Geopolitical affiliation is often referred to as nationality. Functional affiliation may refer to anyone of multinational, political, terrorist, exercise, or other user-defined categories [7].

The OBJECT-ITEM-CAPABILITY class holds the perceived value of a specific CAPABILITY of an OBJECT-ITEM. A CAPABILITY defines or specifies a diverse range of potential abilities to do work, perform a function or mission, achieve an objective, or provide a service, such as maximum speed or maximum storage 
capacity. CAPABILITY is associated with the ACTION-REQUIRED-CAPABILITY class in order to state the required capability of OBJECT-ITEMs when they are needed as resources for carrying out ACTIONs [2]. The ACTION class represents an activity, or the occurrence of an activity that may use resources and may be focused against an objective. ACTION-EVENT and ACTION-TASK are the subclasses of $A C T I O N$. The ACTION-EVENT class is intended to capture ACTIONs that simply occur and need to be noted for which planning is not known. For example, a unit may report its current activity as an event, or an observer in the field could use the $A C T I O N-E V E N T$ class to report his/her sightings. The ACTION-TASK class are ACTIONs for which control can be exercised or are predicted, such as friendly operations, and those enemy activities that are being anticipated as a result of intelligence assessment. The $A C T I O N-T A S K$ specification supports plans, orders, and requests.

The OBJECT-ITEM-LOCATION class links an instance of OBJECT-ITEM to a specific LOCATION. The LOCATION class represents the specification of position and geometry with respect to a specified horizontal frame of reference and a vertical distance measured from a specified datum. Examples of LOCATIONs are point, sequence of points, polygonal line, circle, rectangle, ellipse, fan area, polygonal area, sphere, block of space, and cone. The LOCATION specifies both location and dimensionality [2].

The OBJECT-ITEM-STATUS class represents the perceived condition of a specific $O B J E C T$-ITEM as determined by the reporting organisation, indicating whether a specific OBJECT-ITEM has been booby-trapped (Yes, No or Unknown), and the emission control status of a specific OBJECT-ITEM (Electronic silence, Radio silence and Normal operations) [7]. Situation awareness require knowledge of the status of various objects (past, present, or predicted) to capture administrative, medical, physical, and procedural states or conditions [2].

\section{Analysis of the Integration}

The summarized results of the integration between JC3IEDM OWL constructs and SAW-CORE constructs in our context of situation awareness in C4ISR systems, which we cannot discuss in detail for reason of space, are provided in Table 1.

\section{Context in Situation Awareness}

First, the elementary and core concept in the integration model is a situation. As mentioned earlier, the jc3:CONTEXT-ELEMENT ${ }^{6}$ construct, which is part of jc3:CONTEXT, contains information about all jc3:REPORTING-DATAs that are part of a specific operation or mission. Therefore, the saw:Situation maps to the jc3:CONTEXT-ELEMENT construct enabling operators to obtain a complete integration model for situation awareness. In this context, the saw:DynamicSystem construct represents the part of the C4ISR system responsible of implementing the jc3:REPORTING-DATA construct and the related information.

${ }^{6}$ From here on, constructs from the JC3IEDM OWL ontology are prefixed with 'jc3:', and constructs from the SAW-CORE ontology are prefixed with 'saw:' 
Table 1. Representation mapping analysis of the JC3IEDM OWL and SAW-CORE integration model

\begin{tabular}{|l|l|}
\hline SAW-CORE Construct & \multicolumn{1}{|c|}{ JC3IEDM OWL Construct } \\
\hline Situation & CONTEXT-ELEMENT \\
\hline Goal & $\begin{array}{l}\text { ACTION-OBJECTIVE-ITEM-MARKING, CANDIDATE- } \\
\text { TARGET-LIST and CANDIDATE-TARGET-LIST- } \\
\text { ASSOCIATION }\end{array}$ \\
\hline Relation & $\begin{array}{l}\text { OBJECT-ITEM-ASSOCIATION, HOLDING, OBJECT- } \\
\text { ITEM-OBJECT-TYPE-ESTABLISHMENT and OBJECT- } \\
\text { TYPE-ESTABLISMENT }\end{array}$ \\
\hline Rule & Business rules \\
\hline RelationSymbol & No direct mapping \\
\hline SituationObject & OBJECT-ITEM \\
\hline PhysicalObject & $\begin{array}{l}\text { FACILITY-TYPE, FEATURE-TYPE, MATERIEL-TYPE, } \\
\text { ORGANISATION-TYPE, PERSON-TYPE, FACILITY, } \\
\text { FEATURE, MATERIEL, ORGANISATION, PERSON }\end{array}$ \\
\hline Attribute & $\begin{array}{l}\text { OBJECT-ITEM-AFFILIATION, OBJECT-ITEM- } \\
\text { CAPABILITY, OBJECT-ITEM-LOCATION and OBJECT- } \\
\text { ITEM-STATUS }\end{array}$ \\
\hline Volume & Included in the LOCATION specification \\
\hline Velocity & Included in the LOCATION specification \\
\hline Position & Included in the LOCATION specification \\
\hline AttributeSymbol & No direct mapping \\
\hline PropertyValue & No direct mapping \\
\hline EventNotice & ACTION-EVENT \\
\hline DynamicSystem & No direct mapping \\
\hline & \\
\hline
\end{tabular}

\section{Objects in Situation Awareness}

The saw:PhysicalObject maps to a number of JC3IEDM OWL constructs: jc3:FACILITY-TYPE, jc3:FEATURE-TYPE, jc3:MATERIEL-TYPE, jc3:ORGANISATION-TYPE and jc3:PERSON-TYPE. Since each object has a location in the JC3IEMD-OWL Ontology, we consider all the JC3IEDM objects as physical objects (i.e., according to the SAW-CORE Ontology, objects that defines the volume, position and velocity of a specific object). In this way, saw:PhysicalObjects can take advantage of the instance information associated with jc3:OBJECT-TYPEs, such as jc3:OBJECT-ITEM-LOCATION and jc3:OBJECT-ITEM-CAPABILITY, increasing good decision-making.

The saw:Attribute construct maps to the next JC3IEDM OWL constructs: jc3:OBJECT-ITEM-AFFILIATION, jc3:OBJECT-ITEM-CAPABILITY, jc3:OBJECTITEM-LOCATION and jc3:OBJECT-ITEM-STATUS. These are the attributes that we 
use in our context of situation awareness in C4ISR, which provide a complete and a valid view of a specific situation.

\section{Properties in Situation Awareness}

The saw:PropertyValue construct although it does not have a direct mapping to any JC3IEDM OWL construct, it is related to the next values from $j c 3$ : ReportingDataCategoryCode: 'PLAN-6' (Planned), 'PRDCTD' (Predicted) and 'REP-1' (Reported). Therefore, saw:PropertyValue could be integrated into the jc3:REPORTING-DATA construct in order to obtain additional information about attributes and events related to the situation under study.

\section{Objectives in Situation Awareness}

The saw:Goal maps to two JC3IEDM OWL constructs: jc3:ACTION-OBJECTIVEITEM-MARKING and jc3:CANDIDATE-TARGET-LIST. Recall that the situational awareness is the basis for planning processes in operations that lead to the achievement of objectives.

\section{Relationships in Situation Awareness}

The saw:Relation maps to four JC3IEDM OWL constructs that represent relationships: jc3:OBJECT-ITEM-ASSOCIATION, jc3:HOLDING, jc3:OBJECT-ITEMOBJECT-TYPE-ESTABLISMENT and jc3:OBJECT-TYPE-ESTABLISHMENT. These complementary relations enrich situation awareness with additional information of objects and instances involved in a specific situation. As described earlier, the saw:Relation construct is defined by saw:Rule constructs. The saw:Rule construct maps to the JC3IEDM business rules that are included in [11], and which can be modelled using the Semantic Web Rule Language (SWRL) [12]. For example, the next rule shows how the system can suggest the assignment of resources to actions that have to be performed by a specific situation:

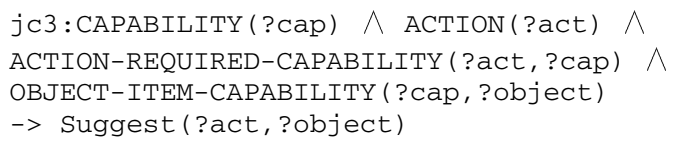

\section{Events in Situation Awareness}

The saw:EventNotice construct maps to the jc3:ACTION-EVENT construct. The jc3:ACTION-EVENTs may trigger jc3:ACTION-TASKs. For example, depending on the jc3:OBJECT-ITEM-HOSTILITY-STATUS of a target, a set of alarms will be triggered in order to manage a specific situation. In the JC3IEDM model, candidate target lists and individual candidate targets can be associated with ACTION-TASKs at the entire list level and at an individual item or type that is being nominated for consideration in the operational planning process.

\section{Conclusions and Outlook}

In this paper, a fusion of the SAW-CORE Ontology and the JC3IEDM OWL Ontology is proposed as a mechanism for improved the generation and sharing of situation 
awareness in C4ISR systems. Using the mapping results summarized in Table 1, the high-level information fusion and enhanced Situation Awareness as well as increase operator performance can be exploited in C4ISR systems including decision making systems. In addition, the ontology-based integration enables operators to elaborate perceptual and cognitive tasks that enhance their decision-making capabilities. The lack of mapping of a SAW-CORE construct to a JC3IEDM OWL construct is not relevant to the integration between these two ontologies, since SAW-CORE constructs could be easily added to the JC3IEDM OWL domain by defining relations to the JC3IEDM OWL constructs that have been mapped.

It is important to note that the most relevant information for building the situation and awareness is covered as for instance the information exchanged by means of the jc3:REPORTING-DATA construct. Considerable amount of information about an operational situation consists of reports by persons or organisations. These generally refer to dynamic data, such as location, status, holdings, associations, and classification, regardless of whether the information refers to friendly, neutral, or hostile elements. It is also important to know for each report the source, the effective and reporting datetimes, and the degree of validity of information. If information is provided without an indication of the source, the validity, and the applicable times, it raises questions as to the source (Who says so?), the quality (Is this information verified?), and timing (When did it happen and when was this reported?). The model can capture both types of information: the substantive information is represented in numerous entities and the amplifying reporting information in the jc3:REPORTINGDATA structure. The jc3:REPORTING-DATA structure provides a mechanism for maintaining a time record that applies not only to the historical past and the immediate present, but also to the future.

Interoperability is also a very important issue for C4ISR systems. On the operational level, interoperability is the ability of a technical system, agency, or person to share information via voice and data signals to another system, agency, or person on demand, in real time, when needed, and as authorized. Regarding interoperability, one of the main achievements of this proposal is to achieve vertical interoperability to ensure situational awareness knowledge spread. Vertical interoperability it is assured by means of a MIP solution, which involves the use of JC3IEDM OWL Ontology since the JC3IEDM is an accepted and established military standard with large support from different nations. Adopting this solution implies that, through this proposal, command and control systems could exchange information among them and among systems based on the SAW-CORE Ontology.

In addition, it is worth noting the duality of the approach since the fusion of these two ontologies could also be applied to civil scenarios, such as Emergency Management Systems (EMS), where the key to efficient and safe cooperation among security forces is to share a jointly maintained, accessible and reliable situation awareness. However, our approach has some limitations:

- In the JC3IEDM OWL Ontology there are two high-level object classes, jc3:OBJECT-TYPE and jc3:OBJECT-ITEM, which are not considered in the SAW-CORE Ontology. The jc3:OBJECT-TYPE construct is derived from an OBJECT-TYPE of the JC3IEDM where is defined as "An individually identified class of objects that has military or civilian significance." It is a generalisation of 
five other object classes that are treated in the data model as subtypes of OBJECT-TYPE (Facility, Feature, Person, Materiel and Equipment). It is used for more generic information associated with a specific class of objects, whereas the jc3:OBJECT-ITEM construct is used to capture information specific to individuals (e.g., height, length, width, major building type...). However, the representation of instances as classes in the JC3IEDM OWL Ontology can difficult the definition of rules and specific class constraints in the fusion ontology.

- On the other hand, the SAW-CORE Ontology lacks of spatio-temporal relation types, which can be solved with the fusion of the two ontologies. In this sense, the SAW-CORE Ontology can be considered as a good starting point in order to extent the information of situation awareness ontologies.

In our future work, we intend to integrate other foremost ontologies to the JC3IEDM OWL Ontology at different levels of the JCL data fusion model in order to improve different C4ISR systems functionalities. Other possible future work that could be tackled is to study a possible extension of the SAW-CORE Ontology and its fusion with the JC3IEDM OWL Ontology to cover new concepts for improving situation awareness.

\section{Acknowledgements}

This work is funded by AMPER Programas through Integra project (CDTI, Spanish Ministry of Science and Innovation).

\section{References}

1. Steinberg, A.N., Bowman, F.L., White, F.E.: Revisions to the JDL Data Fusion Model. In: Joint NATO/IRIS Conference Proceedings, and in Sensor Fusion: Architectures, Algorithms, and Applications. Proceedings of the SPIE, vol. 3719, pp. 330-441 (1999)

2. MIP: The Joint C3 Information Exchange Data Model Overview. Version 3.0.2 (2009), https://mipsite.1sec.dnd.ca

3. Matheus, C.J., Kokar, M.M., Baclawski, K.: A Core Ontology for Situation Awareness. In: Proceedings of the Sixth International Conference on Information Fusion, pp. 545-552 (2003)

4. Matheus, C., Ulicny, B.: On the Automatic Generation of an OWL Ontology Based on the Joint C3 Information Exchange Data Model. In: 12th International Command and Control Research and Technology Symposium (2007)

5. Endsley, M.R.: Design and evaluation for situation awareness enhancement. In: Proceedings of the Human Factors Society 32nd Annual Meeting, pp. 97-101 (1988)

6. Endsley, M.R.: Theoretical underpinnings of situation awareness: a critical review. In: Situation Awareness Analysis and Measurement. Lawrence Erlbaum Associates, Mahawah (2000)

7. Simiatis, E.: Joint C3 Information Exchange Data Model (JC3IEDM) Training Presentation. Part I - General Introduction. Institute for Defense Analyses: Draft Final, IDA Document D-3532 (2007), http://mda.ida.org/CIO-G-6-Deliverables / d-3532/d-3532. document.pdf 
8. MIP: Joint C3 Information Exchange Data Model (JC3IEDM) 3.1d edn., Unclassified (2008)

9. Smith, M.K., Welty, C., McGuinness, D.L.: OWL Web Ontology Language Guide. W3C Recommendation (2004), http: / / www .w3 . org/TR/owl-guide/

10. Matheus, C.J., Baclawski, K., Kokar, M.M., Letkowski, J.J.: Using SWRL and OWL to Capture Domain Knowledge for a Situation Awareness Application Applied to a Supply Logistics Scenario. In: Adi, A., Stoutenburg, S., Tabet, S. (eds.) RuleML 2005. LNCS, vol. 3791, pp. 130-144. Springer, Heidelberg (2005)

11. Annex G2, I.D.A.: Compendium Of Coded Business Rules. MIP Support, Online Documentation, US Army JC3IEDM 3.0.2,

http://mda.ida.org/US-JC3IEDM_302/index.html

12. Horrocks, I., Patel-Schneider, P.F., Boley, H., Tabet, S., Grosof, B., Dean, M.: SWRL: A Semantic Web Rule Language Combining OWL and RuleML. W3C Member Submission (2004), http: / /www.w3 .org/Submission/SWRL/ 